\title{
Stochastic, empirically-informed model of landscape dynamics and its application to deforestation scenarios
}

\author{
J. Nowosad ${ }^{1,2}$, T. F. Stepinski ${ }^{1}$ \\ ${ }^{1}$ Space Informatics Lab, Department of Geography and GIS, University of Cincinnati, Cincinnati, OH \\ ${ }^{2}$ Institute of Geoecology and Geoinformation, Adam Mickiewicz University, Poznan, Poland

\section{Key Points:} \\ - Long-term evolution of an individual landscape is stochastically modeled using a \\ worldwide dataset of short-term landscape changes. \\ - Model suggests that heterogeneous landscapes are transitional stages between ho- \\ mogeneous landscapes of different themes. \\ - In a transit from forested to agricultural landscapes, losing the first $50 \%$ of the \\ forest is slower than losing the rest of the forest.
}

Postprint accepted for publication in Geophysical Research Letters

DOI: https://doi.org/10.1029/2019GL085952

Corresponding author: T. F. Stepinski, stepintz@uc.edu 


\begin{abstract}
Land change including deforestation undermines the sustainability of the environment. Using data on 1992-2015 pattern change in over 1.7 million meso-scale landscapes worldwide we developed a stochastic model of long-term landscape dynamics. The model suggests that observed heterogeneous landscapes are short-lived stages in a transition between quasi-stable homogeneous landscapes of different themes. As a case study we used Monte Carlo simulations based on our model to derive a probability distribution for evolutionary scenarios of landscapes that undergo a forest-to-agriculture transit, a prevalent element of deforestation. Results of simulations show that most likely and the fastest deforestation scenario is through the sequence of highly aggregated forest/agriculture mosaics with a decreasing share of the forest. Simulations also show that once forest share drops below $50 \%$ the remainder of the transit is rapid. This suggests that possible conservation policy is to protect meso-scale tracts of land before the forest share drops below $50 \%$.
\end{abstract}

Plain Language Summary Land change across the world undermines the sustainability of the environment. Understanding the dynamics of landscape change would help to find trade-offs between the development and sustainability of the environment. We developed a data-driven model capable of providing plausible scenarios of long-term evolution of landscapes. Using this model, we proposed a general principle of landscape evolution: heterogeneous landscapes are short-lived stages in transit between two homogeneous landscapes of different land cover. As a case study we applied our model to identify the most likely scenarios of the forest-to-agriculture transit, a dominant source of deforestation. The model suggests that scenarios of the forest-to-agriculture transits that proceed through a sequence of aggregated mosaics are more frequent and more damaging to an environment than transits proceeding through a sequence of disaggregated mosaics. It also suggests that preserving meso-scale $\left(\sim 100 \mathrm{~km}^{2}\right)$ tracts of land before they lose $50 \%$ of forest share may be a good conservation strategy.

\title{
1 Introduction
}

Earth's lands are changing due to anthropogenic impact (Venter et al., 2016). Examples of change due to a direct human impact include urbanization and deforestation. Desertification provides an example of change due to an indirect human impact via climate change. Remote sensing of the land surface from space over the last few decades reveals that land change is a globally significant environmental trend (Song et al., 2018) that affects most landmasses and land themes with the deforestation of tropical forests being its most pronounced example (Hansen et al., 2013). Understanding the long-term dynamics of land changes is needed to identify policy options aimed at mitigating its negative impacts (Chhabra et al., 2006).

Currently, landscape dynamics is addressed in two contexts; (1) in remote sensing (Verburg et al., 2004; Liu \& Yang, 2015; Olmedo et al., 2018) land use/cover change (LUCC) models provide short-time predictions of land use/cover change, and (2) in landscape ecology (Gaucherel \& Houet, 2009; Gaucherel et al., 2014) landscape models (LM) predict how change in landscape pattern influences the ecological process. LUCC models (NRC, 2014; Mas et al., 2014) are designed to predict land change mostly on a pixel-by-pixel basis though some polygonal or patch-based models have also been proposed (Meentemeyer et al., 2013; Xu \& Brown, 2017). Most LUCC models investigate the land change in a specific type of landscape (for example, agricultural, forested, arid, or urban) in the local study areas, although some regional-scale LUCC models have also been published (deNijs et al., 2004; Soares-Filho et al., 2006; Tayyebi et al., 2013). LMs are designed (Gaucherel et al., 2014) to either simulate a specific process which causes a change to landscape pattern (see, for example, Pe'er et al. (2013)) or to use a simple set of arbi- 
trary rules (so-called neutral LM model) to simulate a progression of artificial landscapes having patterns and statistical properties similar to observed landscapes.

Our interest is in understanding a long-term evolution of landscapes which results in a profound change to the land use/cover over large spatial extents. One example of such evolution is the forest $\rightarrow$ agriculture transit which leads to deforestation. What are the most likely intermediate steps of such a transit? Are some evolutionary paths more likely than others? Neither LUCC models nor LMs can address these questions.

To be able to start to address such problems we have developed a stochastic, empirically informed model of landscape dynamics. This model is unlike either LUCC models or LMs. It is based on the principle that a probability distribution of long-term trajectories of a single landscape can be constructed from the frequencies of various changes to a large number of landscapes during a single short-term period. The basic spatial unit of our analysis is a meso-scale ( $\sim 100 \mathrm{~km}^{2}$ area) landscape (a pattern of land cover classes). To gather the frequencies of short-term changes we divided the entire terrestrial landmass area into $\sim 1,700,000$ such landscapes and tabulated their (short-term) changes between 1992 and 2015. Landscapes and their transitions are classified into a finite number of types thus enabling calculation of probabilities that a given landscape type changes to another landscape type over the period of 23 years. Assuming that, in the first approximation, the set of transition types and their frequencies is complete and stationary, the set of transition probabilities constitutes a stochastic, empirically-informed model of landscape evolution that can be used to address questions like those asked in the previous paragraph.

The major purpose of this paper is to describe the stochastic, empirically informed model of landscape dynamics. In addition, we demonstrate the working of the model by simulating the evolution of the landscape from forested to agricultural. Starting from a landscape of homogeneous forest and ending with a landscape of homogeneous agricultural land, the simulation yields different deforestation scenarios and their probabilities. A landscape currently covered entirely by forest will most likely evolve to an agricultural landscape along the maximum likelihood trajectory which reflects a prevailing series of circumstances; this is the most likely deforestation scenario. For a forest $\rightarrow$ agriculture transit to happen along less likely trajectories rare circumstances will have to happen; these are less likely deforestation scenarios.

\section{Data and Methods}

We conceptualize land change in a given areal unit as a modification of landscape pattern within this unit between two times of observations. As an input for our model we use the European Space Agency (ESA) Climate Change Initiative (CCI) global dataset (CCI-LC) at $t_{0}=1992$ and at $t_{1}=2015$ (ESA, 2017). CCI-LCs are temporally consistent, 300m-resolution maps of 22 land cover classes, which we have reclassified into nine broader Intergovernmental Panel on Climate Change (IPCC) land categories (see the legend to Fig. 1A). The entire terrestrial landmass is tessellated into 1,764,740 nonoverlapping square areal units of the size $9 \mathrm{~km} \times 9 \mathrm{~km}(30 \times 30$ CCI-LC cells $)$. A mosaic formed by the nine land categories in a given areal unit at a given time constitutes a meso-scale landscape (hereafter referred to as a landscape).

A landscape is characterized by its configuration (a geometry of its pattern) and its thematic content (names of land cover classes present). Landscape configuration can be succinctly parametrized (Nowosad \& Stepinski, 2018) by only two metrics interpreted as "complexity" $C$ and "aggregation" $A$. The land cover data is transformed into a database consisting of $1,764,740$ landscapes having attributes pertaining to landscape thematic composition $\left\{\rho_{1}^{t}, \ldots, \rho_{9}^{t}\right\}$ and landscape configuration $\left\{C^{t}, A^{t}\right\}$, where $t=1992$ or 2015 . 

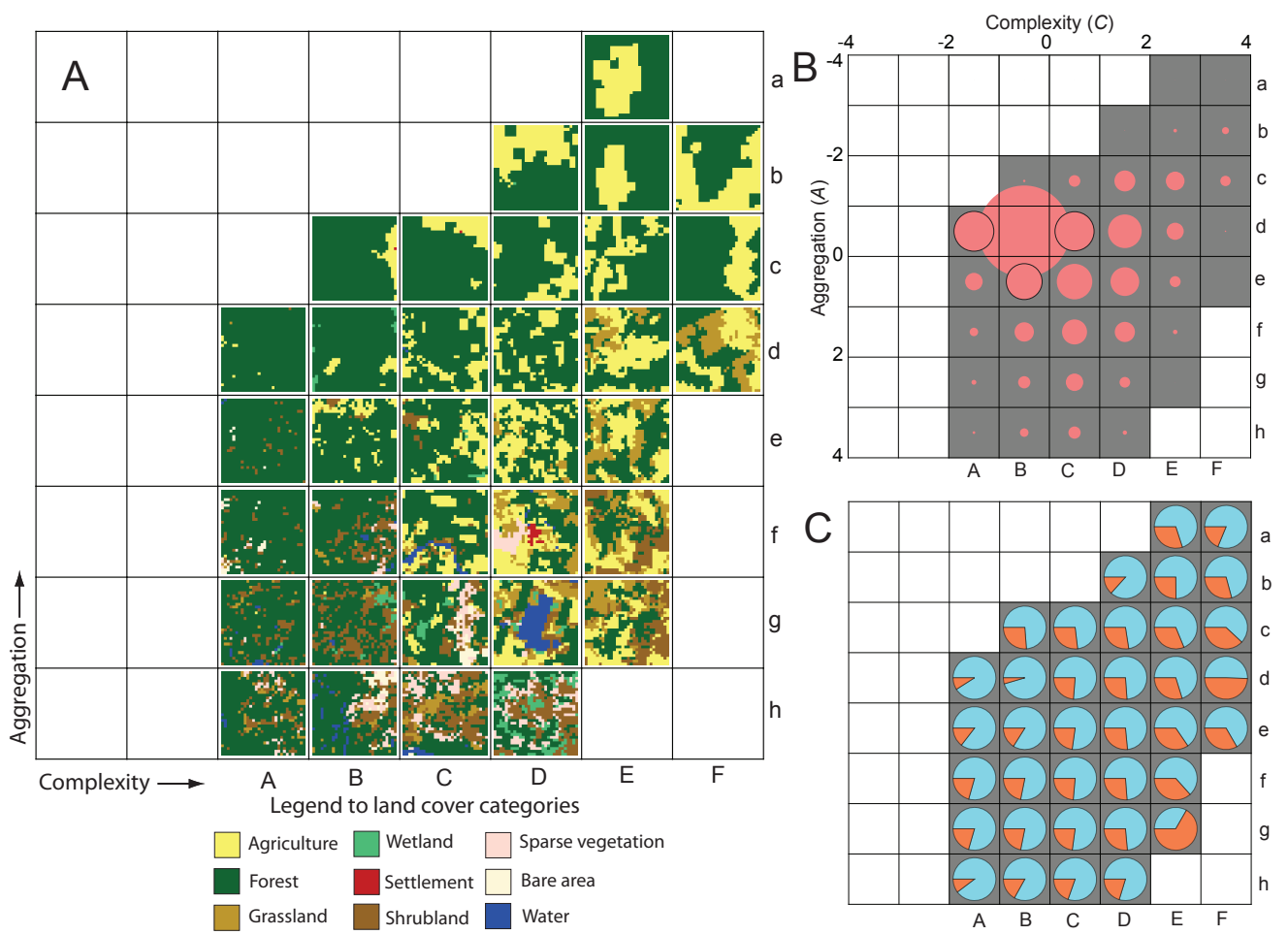

Figure 1. (A) Archetypes of pattern configurations of worldwide landscapes organized on the $C-A$ diagram along increasing complexity (left-to-right) and aggregation (bottom-to-top). To better visualize the progression of patterns the archetypes are chosen only from among landscapes with a forest as the top land cover category. (B) A histogram of all landscapes at $t_{0}$ with bins corresponding to landscape types organized into the $C-A$ grid (diagram). Empty cells in the $C-A$ grid correspond to landscape pattern configurations that are theoretically possible but not present in the dataset. Gray-colored entries indicate non-zero bins. Areas of red circles are proportional to the fraction of landscapes in the bin; the sum of all bins equals to 1. (C) Probability of landscapes of a given type to maintain its type (blue) or to transition to another type (red) during the period $\Delta t$. 
To simplify the dataset we classified all landscapes into up to 64 classes with respect to their configurations (see Nowosad \& Stepinski (2018) for details). This is a 2D classification that takes into consideration values of $C$ and $A$ but not landscape's thematic content (Computational details are given in the supporting information file, the flow of computations is outlined in Fig. S1.) Landscapes of similar values of $C$ and $A$ belong to the same class regardless of their thematic content. Fig. 1A shows exemplars of landscape types taken from a forest-theme subset of all landscapes; only 34 landscape types are present in this subset. Sets of archetypes, selected from different theme subsets of landscapes are shown in supporting information (Fig. S2). Note that it is convenient to show the archetypes as well as all other results in a grid corresponding to increasing complexity and aggregation of landscapes. We refer to such a grid as the $C-$ $A$ diagram. This diagram systematizes landscape types. From left to right the landscapes are generally more complex (they contain more land cover categories). From bottom to top the landscapes are generally more aggregated (they have larger patches).

Fig. 1B shows a normalized histogram for all classified landscapes at $t_{0}$. Note that 36 out of 64 theoretically possible landscape types are present in the dataset. From Fig. 1B it is clear that the $\mathrm{Bd}$ type, which corresponds to a "simple" (low complexity) landscape is the most frequent ( $42 \%$ of all landscapes). Note that landscapes with disaggregated and/or more complex configurations tend to be less abundant. Fig. $1 \mathrm{C}$ pertains to the stability of landscape configurations during the period of $\Delta t=t_{1}-t_{0}=23$ years. $\mathrm{A}$ landscape is stable if during $\Delta t$ it did not change its type. A red part of a pie diagram shows the probability of a landscape of a given type to transition to another type during $\Delta t$, and the blue part shows the probability of a landscape to maintain its type. The most stable type is again Bd while other landscape types are less stable. There is an association between landscape type's abundance and its stability (correlation coefficient equal to 0.6) - more abundant landscape types are more stable. Fig. S3 (in supporting information) shows histograms and stability grids (equivalents of Fig. 1B and Fig. 1C) calculated for different theme subsets of all landscapes.

Overall, landscape statistics (illustrated in Figs. 1,S2,S3) suggest that a near homogeneous configuration is the most abundant and the most stable landscape pattern for six out of eight themes considered. An exception is the subset of majority-urban landscapes and wetland landscapes.

\section{Transitions}

A transition has occurred when a landscape changed its type to another type during the period $\Delta t$. Among the 1,764,740 landscapes in our database, only 257,031 (15\%) had transitioned during $\Delta t$. Because there is a finite number of landscape types there is also a finite number of possible transitions. Fig. 2A illustrates a transition matrix calculated from 1992-2015 transitions of 257,031 landscapes. The transition matrix is organized into the $C-A$ diagram. First, consider the part of the matrix corresponding to transitions from the type Dc, this part is shown in detail in Fig. 2B. During $\Delta t$ a fraction of Dc landscapes transitioned to 20 (out of possible 64) different types indicated by reddish colors. Darker shades of red indicate more transitions; the most frequent transition is Dc $\rightarrow$ Bd. Similarly, other parts of the transition matrix show the frequencies of transitions from other landscape types.

Given our assumption of completeness and stationarity of transition types (see the introduction), Fig. 2A depicts a stochastic model of landscape evolution based on actual observations. A given landscape at time $t_{n}$ is first generalized to its type. The stochastic model assigns a type to this landscape at $t_{n+\Delta t}$ with probability indicated by the transition matrix. Repeating this procedure multiple times (at each time the model would select a transition in accordance with the corresponding probability distribution) we ob- 

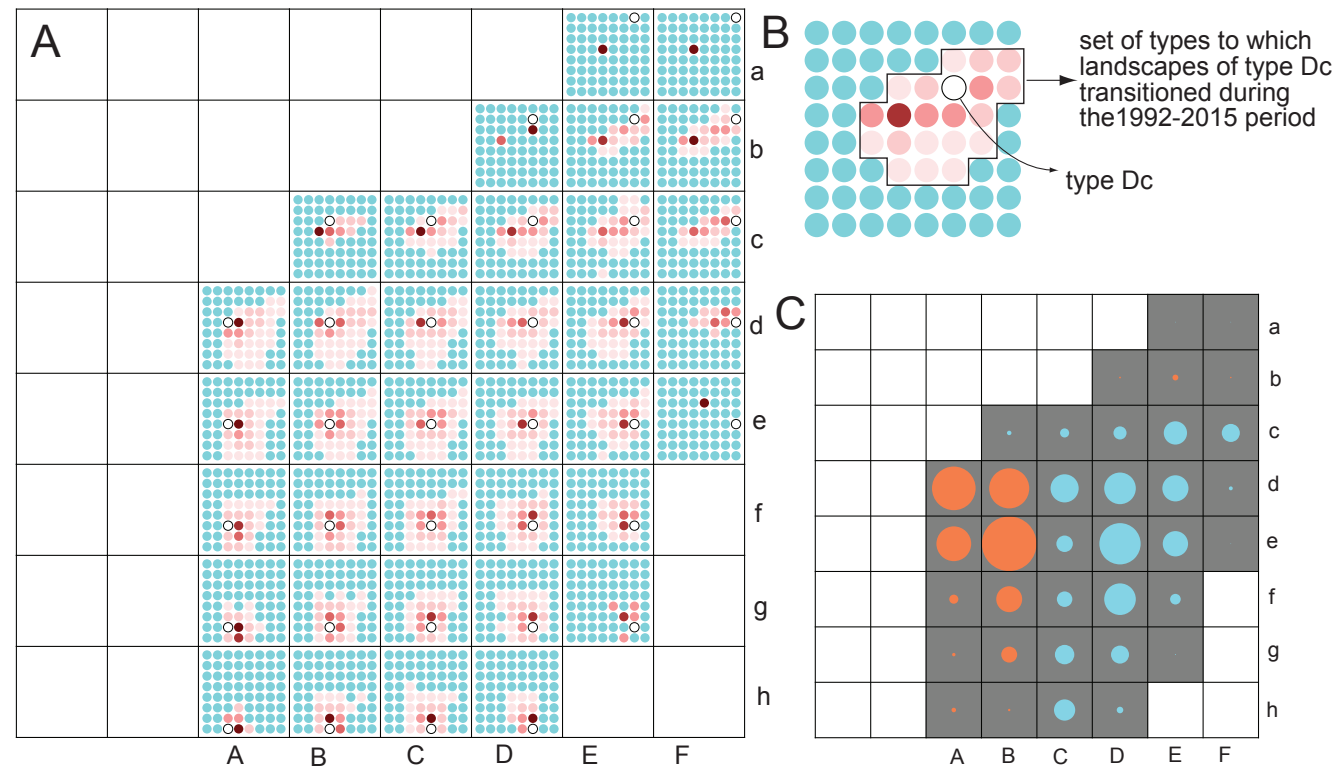

Figure 2. (A) Visualization of the transition matrix. The whole matrix is divided into smaller parts $(8 \times 8$ matrices) corresponding to transitions from specific landscape types; these parts are organized in the $C-A$ grid. (B) A magnification of a single part of the transition matrix corresponding to the landscape type Dc (shown by the white circle) at $t_{0}$. Reddish-colored circles indicate landscape types to which landscape of type Dc transitioned during $\Delta t$; the darker the color the larger the probability of transition. (C) Diagram illustrating a net change to the histogram of landscape types during $\Delta t$. Areas of circles are proportional to the magnitude of the net change, red color indicates the gain and blue color indicates the loss. 
tain a possible evolutionary trajectory of the landscape. A large number of such simulations yield the probability distribution function of future trajectories.

From Fig. 2A we observe that most landscape types may evolve in many different ways (in terms of changes to values of complexity and aggregation metrics), but with different probabilities. For example, types located at the edges of the $C-A$ diagram must evolve away from those edges. Combining information from Fig. 2A with information from Figs. $1 \mathrm{~B}$ and $\mathrm{C}$ it follows that landscapes of type $\mathrm{Bd}$ (which are almost homogeneous) are long-lived stable configurations, whereas other landscapes are relatively shorterlived and thus less stable configurations. Note that this is a purely empirical finding whose explanation is beyond the scope of this paper.

Fig. 2C illustrates the change to the histogram of landscape configurations (shown in Fig. 1B) after $\Delta t$. This change is the net result of all 257,031 transitions. As landscapes transition in and out of different types, the net change amounts to only 20,408 landscapes, which, as can be seen in Fig. 2C, results in some (relatively small) increase in the counts for bins corresponding to simpler landscapes at the cost of a decrease in the counts for the remaining bins. Fig. S4 (in supporting information) shows transition matrices and graphs of histogram changes (equivalents of Fig. 2A and Fig. 2C) calculated for subsets of landscapes selected to have the same top category of land cover. Overall, the results for the thematic subsets of landscapes follow the same pattern as the results for all landscapes. Notable exceptions are majority-urban landscapes, where transition probability distributions are all skewed to the left of the $C-A$. This is consistent with the process of urbanization when landscapes which are already majority-urban increase the urban share of the landscape and become configurationally simpler. Other exceptions are majority-forest landscapes, where a change to the histogram reveals the loss of simple, Bd-type landscapes and the increase in bi-thematic landscapes (for example Dc-type) and forested landscapes with an increasing number of intrusions (for example, Bf-type). This is consistent with significant deforestation of tropical forests during $\Delta t$.

\section{Deforestation scenarios}

Deforestation is the most pronounced example of human-caused landscape change. In most cases, the forest is replaced by agricultural land cover (we refer to this long-term change as the forest $\rightarrow$ agriculture transit or FAT). We envision a FAT as a sequence of transitions between subsequent landscape types starting with a fully forested landscape and ending with a fully agricultural landscape; this sequence is referred to as a trajectory. We use our stochastic model to simulate a probability distribution function of the FAT trajectories. Each FAT trajectory can be divided into two phases, a forest-dominant phase (FAT1), and an agriculture-dominant phase (FAT2). To build a model for FAT1 we use a subset of all landscapes subject to the following conditions: forest and agriculture are the primary and secondary land cover classes in 1992 and 2015; 1992-2015 transitions are toward a diminishing share of the forest. To build a model for FAT2 we use a subset of all landscapes subject to the following conditions: agriculture and forest are the primary and secondary land cover classes in 1992 and 2015; 1992-2015 transitions are toward an increasing share of agriculture. Fig. S5 (in supporting information) shows landscape pattern types archetypes selected from among landscapes selected for the two phases of the models and their transition matrices.

We simulate FAT trajectories using the Monte Carlo method (Rubinstein \& Kroese, 2016). The simulation starts from almost totally forested landscape (type Ad). At each step, a transition from a current stage to the next is chosen randomly according to a probability distribution of transitions as given by the FAT1 part of the model until landscape evolves to type $\mathrm{Fb}$ or Fc. Subsequently, the next evolutionary stage is chosen randomly according to a probability distribution of transitions as given by the FAT2 part of the 

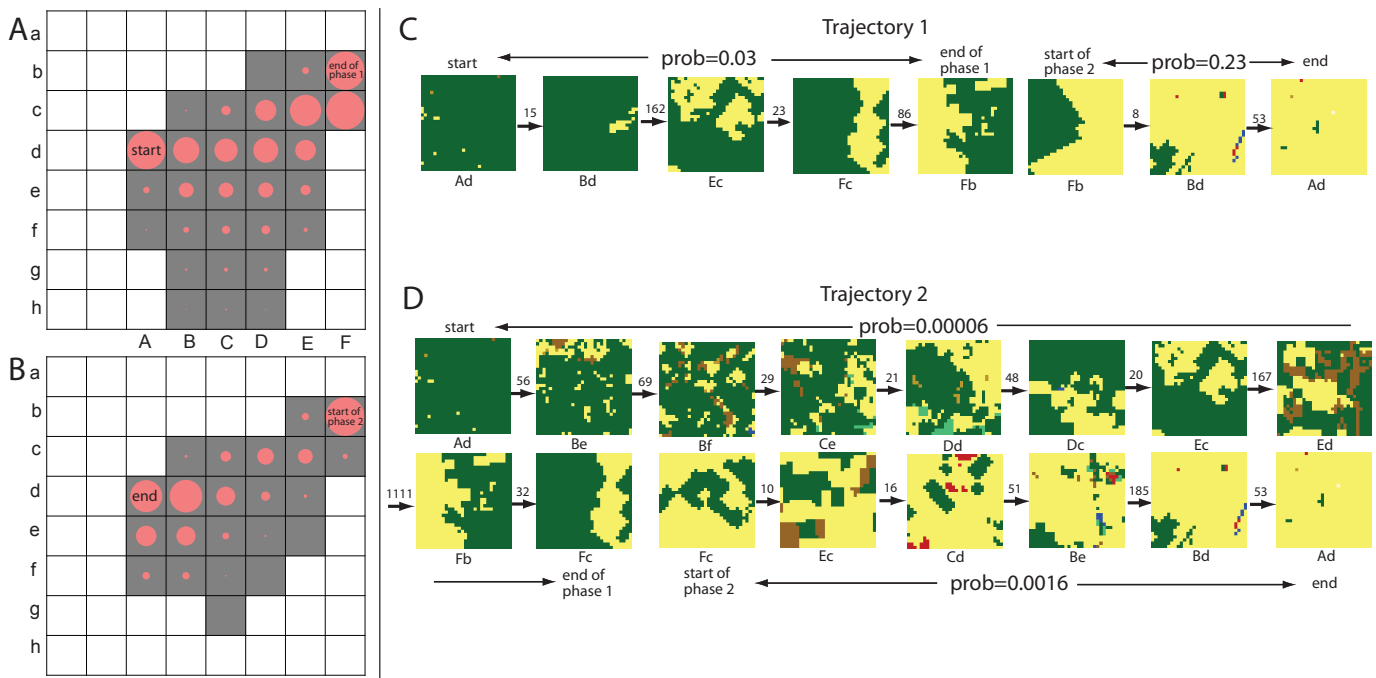

Figure 3. Summary of the forest-dominated phase (A) and agriculture-dominated phase (B) trajectories simulating an evolution from a homogeneous forested landscape to a homogeneous agricultural landscape. The span of all trajectories is restricted to gray-colored landscape types. Areas of red circles are proportional to the fraction of all trajectories passing through a given landscape type. All simulated trajectories start at type Ad. (C) The most likely trajectory. (D) An example of a much less likely trajectory. Numbers placed over the arrows are mean waiting times for transitions between two consecutive stages to occur.

model. The simulation ends when the trajectory reaches one of the landscapes at the left edge of the $C-A$ diagram. Using this procedure we have generated 20,000 FAT trajectories from which a probability distribution function of FAT trajectories is calculated.

Fig. 3A summarizes the first phase and Fig, 3B summarizes the second phase of simulated FATs. Individual trajectories are not shown because a figure showing 20,000 trajectories would be illegible, instead, areas of the red circles in Figs. 3A and B show fractions of trajectories passing through a given landscape type; $100 \%$ of trajectories pass through the type Ad from which all simulations start. High probability trajectories pass only through types with large red circles, whereas low probability trajectories pass also through types with small red circles. According to Figs. 3A and B, high probability trajectories pass through landscape types corresponding to highly aggregated patterns (look for landscape archetypes shown in Fig. S4 corresponding to types frequented by the trajectories). The most likely FAT trajectory is shown in Fig. 3C. This trajectory depicts the forest $\rightarrow$ agriculture transit in eight stages. Fig. 3D shows an example of a less likely FAT trajectory. This trajectory depicts the forest $\rightarrow$ agriculture transit in sixteen stages. The difference between this trajectory and the most likely trajectory is that in both stages the evolution goes through a series of disaggregated landscapes.

Note that the most likely FAT trajectory is not a trajectory where each subsequent stage is determined by the highest transition probability of its proceeding stage as could be expected. Such locally optimal trajectory is $(\mathrm{Ad} \rightarrow \mathrm{Bd} \rightarrow \mathrm{Cd} \rightarrow \mathrm{Dd} \rightarrow \mathrm{Ec} \rightarrow \mathrm{Fc} \rightarrow$ $\mathrm{Fb})+(\mathrm{Fb} \rightarrow \mathrm{Bd} \rightarrow \mathrm{Ad})$ and has half the probability of the most likely trajectory. The difference between this trajectory and the maximum likelihood trajectory is in phase 1, where the maximum likelihood trajectory makes a jump from $\mathrm{Bd}$ to $\mathrm{Ec}\left(p_{\mathrm{tr}}=0.076\right)$ instead of following the locally largest probability $\left(p_{\mathrm{tr}}=0.26\right)$ transition $\mathrm{Bd} \rightarrow \mathrm{Cd}$. This jump saves the maximum likelihood trajectory from visiting stages $\mathrm{Cd}$ and $\mathrm{Dd}$ and, on 
balance, makes this trajectory twice as likely as the trajectory following the largest local transition probability.

A stage-to-stage transition probability of a landscape during $\Delta t$ is a product of a probability $p_{\mathrm{ch}}$ that a landscape would transition to another type (see Fig. 1C) and a probability $p_{\mathrm{tr}}$ of the landscape transitioning to a specific different type (see Fig. S4). Thus, the product $p_{\mathrm{ch}} p_{\mathrm{tr}}$ is a probability of a specific stage-to-stage transition occurring during a single $\Delta t$. The mean waiting time (in units of $\Delta t$ ) for such transition to occur is $1 /\left(p_{\operatorname{ch}} p_{\text {tr }}\right)$.

Numbers placed over the arrows in Figs. 3C and 3D are mean waiting times for transitions between two consecutive stages to occur. For example, a transition $\mathrm{Ad} \rightarrow \mathrm{Bd}$ in the most likely FAT trajectory is $15 \Delta t=345$ years. The key to understanding this value is to remember that it reflects the mean waiting time for such a transition to occur. When considering the entire world, most homogeneous, forested landscapes do not change their pattern type on the time scale of 23 years resulting in $p_{\mathrm{ch}}=0.15$. Thus, despite the $p_{\mathrm{tr}}=0.43$, the total probability of this transition is 0.0645 resulting in the mean waiting time of 345 years. In most cases, high values of the mean waiting time are due to low values of $p_{\mathrm{ch}}$. Analyzing mean waiting times in trajectories shown in Figs. 3C and 3D we observe that phase 1 of FAT trajectories takes longer to complete than phase 2. Thus, it takes longer to lose the first $\sim 50 \%$ of the forest, than it is to lose the remaining forest; the FAT accelerates after reaching a tipping point.

\section{Discussion and Conclusions}

In this paper, we presented a novel methodology for simulating the long-term evolution of landscapes. What distinguishes our approach from existing methods is that it simulates the probability distribution function of long-term trajectories of a single landscape based on observation of 1992-2015 (short-term) transitions for a large number of different landscapes. Thus, using the Monte Carlo method, we can obtain the most likely evolutionary trajectory for a given landscape type. The method is best suited for investigating evolutionary scenarios for specific types of land change, such as deforestation, desertification, or urbanization.

The major advantage of the model is its empirical character. This makes unnecessary to account explicitly for all different processes responsible for the land change as they are implicitly accounted for by the large number statistics using methodology which resembles machine learning. The major shortcoming of the model is that it requires (in the current implementation) some strong assumptions. The first assumption is the temporal stationarity of the transition matrix. The matrix reflects prevailing circumstances of change during the 1992-2015 period and the model assumes that those circumstances do not change. Thus, simulated trajectories reflect stationary scenarios; they show how the landscape would evolve if processes driving the change and their intensities remain unchanged. These are likely to be only the zeroth-order approximations. The second assumption is that our model (as presented in this paper) is built on the worldwide statistics. This has been done so the probabilities are calculated from the largest possible statistics of transitions. However, as a result, transition probabilities are spatially stationary which does not account for variations occurring at the regional level. The assumption of spatial stationarity can be lifted by restricted the data used to construct a model to a specific region at the cost of decreasing the number of observations from which probabilities are inferred.

These assumptions notwithstanding, our model offers an attractive addition to researching landscape dynamics. It is instructive to contrast it with a neutral model (Gaucherel et al., 2014). A neutral model uses an artificial dynamics to produce a series of landscapes with controllable patterns to emulate a specific process - it is useful to obtain specific 
answers to narrowly defined questions (for example, see With (1997)). Our model uses real-world dynamics (implicitly taking into consideration all existing processes) to produce a plausible evolutionary trajectory - it is useful to obtain generalized answers to broadly defined questions. Two examples of our approach answering broad questions were presented in this paper.

First, we were able to address the following broad question: What is the overall principle of landscape evolution? By analyzing statistics of 1995-2015 transitions we proposed the following answer: meso-scale landscapes are predominantly transitional stages in the evolution from a homogeneous, quasi-stable landscape of one land cover class to another homogeneous, quasi-stable landscape of different land cover class. To what degree this hypothesis depends on the stationarity of our model remains unknown, but the model at least allows to consider such a question and to formulate a hypothesis. A corollary of our hypothesis is that homogeneous landscapes are much more abundant than landscapes having complex patterns, which indeed has been shown (Nowosad \& Stepinski, 2018) to be the case.

Second, we were able to address the question of how the forest $\rightarrow$ agriculture transition happens on a meso-scale? By performing Monte Carlo simulations based on our model we derived a probability distribution function for the forest $\rightarrow$ agriculture trajectories. The character of this distribution (see Fig. 3) provides the following answer: the forest $\rightarrow$ agriculture transit is most likely to proceed through a series of intermediate landscapes characterized by highly aggregated forest-agriculture mosaics; once forest share drops below $50 \%$ the remainder of the transit is rapid.

As the times involved in a complete forest $\rightarrow$ agriculture transit on the meso-scale are quite long, direct verification of this hypothesis is not yet possible given that remotely sensed images on the global scale are only available for the past $\sim 30$ years. In this paper, we have concentrated on the introduction of the idea of the stochastic, empiricallyinformed model and we chose the longest period ( $\Delta t=23$ years) available in the CCILC data to maximize the number of transitions from which to build a model. However, future work can investigate models built using $\Delta t=1-5$ years transitions. CCI-LC allows for the construction of multiple such models during the 1992-2015 period; they can be used to check a degree to which the assumption of temporal stationarity is violated, and to check, at least partially, our assertion that the forest $\rightarrow$ agriculture transit is most likely to proceed through highly aggregated stages.

Finally, land change scenarios other than FAT can be studied using our model. It is a matter of selecting an appropriate subset of the dataset. Desertification can be studied by considering grassland $\rightarrow$ sparse, grassland $\rightarrow$ bare, and sparse $\rightarrow$ bare transits. Urbanization can be studied by considering transitions from agriculture, forest, shrub, and sparse to urban. Shrinkage of wetlands can be studied from wetland $\rightarrow$ agriculture transits.

\section{Acknowledgments}

This work was supported by the University of Cincinnati Space Exploration Institute. Database used in this research is available from the Center for Open Science (Nowosad et al., 2019).

\section{References}

Chhabra, A., Geist, H., Houghton, R. A., Haberl, H., Braimoh, A. K., Vlek, P. L., et al. (2006). Multiple impacts of land-use/cover change. In E. F. Lambin \& H. Geist (Eds.), In Land-use and land-cover change (pp. 71-116). Springer, Berlin, Heidelberg. 
deNijs, T. C., DeNiet, R., \& Crommentuijn, L. (2004). Constructing land-use maps of the Netherlands in 2030. Journal of environmental management, 72(1-2), 3542.

ESA. (2017). European Space Agency Land Cover CCI Product User Guide Version 2.0 (Tech. Rep.).

Gaucherel, C., \& Houet, T. (2009). Preface to the selected papers on spatially explicit landscape modelling: current practices and challenges. Ecological Modelling, 220(24), 3477-3480.

Gaucherel, C., Houllier, F., Auclair, D., \& Houet, T. $\quad$ (2014). Dynamic landscape modelling: the quest for a unifying theory. Living Rev. Landscape Res., 8(2), 531 .

Hansen, M. C., Potapov, P. V., Moore, R., Hancher, M., Turubanova, S., Tyukavina, A., et al. (2013). High-resolution global maps of 21st-century forest cover change. Science, 342, .850-853.

Liu, T., \& Yang, X. (2015). Land Change Modeling: Status and Challenges. In Monitoring and Modeling of Global Changes: A Geomatics Perspective (pp. 3-16). Springer, Dordrecht.

Mas, J. F., Kolb, M., Paegelow, M., Camacho-Olmedo, M. T., \& Houet, T. (2014). Inductive pattern-based land use/cover change models: A comparison of four software packages. Environmental Modelling \& Software, 51, 94-111.

Meentemeyer, R. K., Tang, W., Dorning, M. A., Vogler, J. B., Cunniffe, N. J., \& Shoemaker, D. A. (2013). FUTURES: multilevel simulations of emerging urban rural landscape structure using a stochastic patch-growing algorithm. Annals of the Association of American Geographers, 103(4), 785-807.

Nowosad, J., \& Stepinski, T. F. (2018). Global inventory of landscape patterns and latent variables of landscape spatial configuration. Ecological Indicators, 89, 159167.

Nowosad, J., Stepinski, T. F., \& Netzel, P. ～(2019, Jan). Global assessment and mapping of changes in mesoscale landscapes: 1992-2015. OSF. Retrieved from https://osf .io/34g9c

NRC. (2014). Advancing land change modeling: opportunities and research requirements (Tech. Rep.). National Research Council and Geographical Sciences Committee. National Academies Press.

Olmedo, M. T., Paegelow, M., Mas, J. F., \& Escobar, F. (Eds.). (2018). Geomatic Approaches for Modeling Land Change Scenarios. Springer.

Pe'er, G., Zurita, G. A., Schober, L., Bellocq, M. I., Strer, M., Müller, M., et al. (2013). Simple process-based simulators for generating spatial patterns of habitat loss and fragmentation: a review and introduction to the G-RaFFe model. PloS One, 8(5), e64968.

Rubinstein, R. Y., \& Kroese, D. P. (2016). Simulation and the Monte Carlo method. John Wiley \& Sons.

Soares-Filho, B. C., Nepstad, D. C., Curran, L. M., Cerqueira, G. C., Garcia, R. A., Ramos, C. A., et al. (2006). Modelling conservation in the Amazon basin. Nature, 440, 520-523.

Song, X. P., Hansen, M. C., Stehman, S. V., Potapov, P. V., Tyukavina, A., Vermote, E. F., et al. (2018). Global land change from 1982 to 2016 . Nature, 560, 639-643.

Tayyebi, A., Pekin, B. K., Pijanowski, B. C., Plourde, J. D., Doucette, J. S., \& Braun, D. (2013). Hierarchical modeling of urban growth across the conterminous USA: developing meso-scale quantity drivers for the Land Transformation Model. Journal of Land Use Science, 8(4), pp.422-442., 8(4), 422-442.

Venter, O., Sanderson, E. W., Magrach, A., Allan, J. R., Beher, J., Jones, K. R., et al. (2016). Sixteen years of change in the global terrestrial human footprint and implications for biodiversity conservation. Nature Communications, 7, 12558. 
Verburg, P. H., P. Schot p., Dijst, M. J., \& Veldkamp, A. (2004). Land use change modelling: current practice and research priorities. GeoJournal, 61(4), 309-324.

With, K. A. (1997). The Application of Neutral Landscape Models in Conservation Biology. Conservation Biology, 11(5), 1069-1080.

Xu, H., \& Brown, D. G. (2017). Sensitivity of a stochastic land-cover change model to pixel versus polygonal land units. International Journal of Geographical Information Science, 31(7), 738-762. 


\title{
Supporting information for "Stochastic, empirically-informed model of landscape dynamics and its application to deforestation scenarios"
}

\author{
J. Nowosad ${ }^{1,2}$, T. F. Stepinski ${ }^{1}$ \\ ${ }^{1}$ Space Informatics Lab, Department of Geography and GIS, University of Cincinnati, Cincinnati, OH \\ ${ }^{2}$ Institute of Geoecology and Geoinformation, Adam Mickiewicz University, Poznan, Poland
}

\section{Content of this file}

This file contains some additional details on the computational method used in the paper, as well as figures S2, S3, S4, and S5 referred to in the paper.

\section{Details of computational method}

Fig. S1 shows a diagram outlining the consecutive computational steps of our method. These steps are numbered from 1 to 6 . This section provides some additional details for each of these steps.

Step 1. The most computationally demanding part of the calculation is to obtain the global dataset of meso-scale landscapes and to calculate the values of the two metrics, complexity $(C)$ and aggregation $(A)$. These two tasks have been previously described in Nowosad \& Stepinski (2018a) and Nowosad et al. (2019), and the complete dataset is available for download from the Center for Open Science (Nowosad \& Stepinski, 2018b). To obtain landscapes of approximately the same size and shape, the global CCI-LC dataset is reprojected to the Fuller projection and tessellated into $9 \mathrm{~km} \times 9 \mathrm{~km}$ tiles. We used GeoPAT software (Netzel et al., 2018) to achieve this task. Calculation of metrics $C$ and $A$ were preformed in $\mathrm{R}$ (R Core Team, 2019).

Step 2. Metrics $C$ and $A$ are principal components and have a theoretical range of values between -4 and 4 (Nowosad \& Stepinski, 2018a). We classify all landscapes based on their values of $C$ and $A$. This is achieved by constructing an equispaced $C-A$ grid that divides the $C-A$ space into 64 sections, each section having $\Delta C=1$ and $\Delta A=$ 1 ; each section is associated with a different landscape type. Assigning a type to a given landscape is a matter of simply finding to which section in the $C-A$ grid its values of $C$ and $A$ belong. Note that the actual dataset, even as large as the one we are using, may not contain landscapes of all 64 theoretically possible types. Indeed, landscapes in our entire dataset belong to only 36 out of 65 theoretically possible types. Landscapes in subsets of our dataset may belong to an even smaller number of types.

Step 3. Note that a classification of landscapes based on $C$ and $A$ pertains to their geometric configuration but ignores their thematic content. The thematic content is given by landscape composition - fractions of landscape area $\left\{\rho_{1}, \ldots, \rho_{9}\right\}$ occupied by forest, agriculture, urban, wetland, grassland, sparse vegetation, bare land, and water, respectively. For example, for Fig.1A we have selected landscapes such that $\rho_{1}>\rho_{2} \ldots>$ other fractions. If we want to simulate a specific process, for example, a forest-to-agriculture transit, we select only a suitable subset of landscapes based on its composition.

Step 4. A landscape that has a given type in 1992 may have a different type in 2015. Using our dataset, we calculate frequencies of landscape types changes. This re-

Corresponding author: T. F. Stepinski, stepintz@uc.edu 


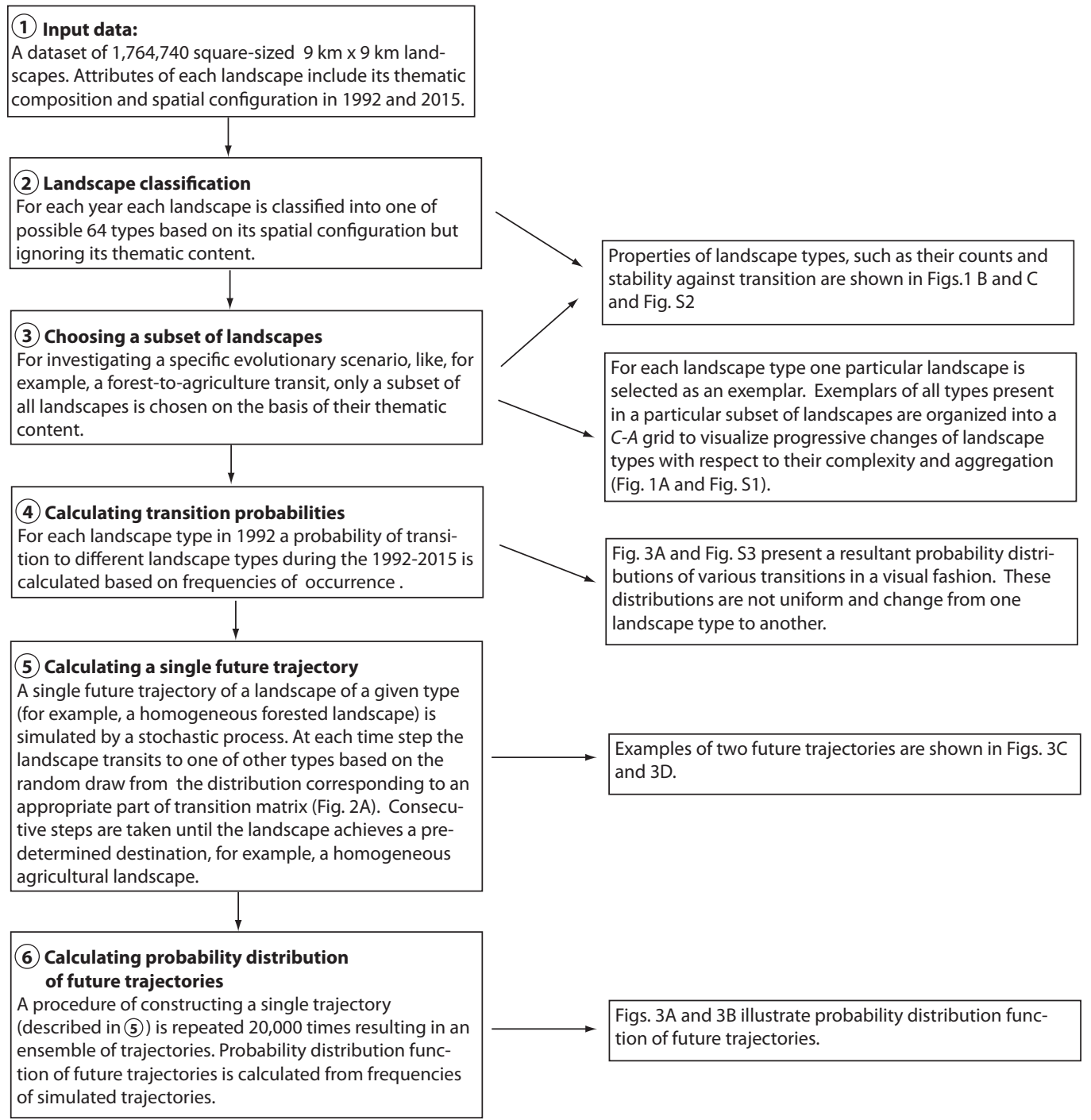

Figure S1. Diagram outlining consecutive computational steps to obtain a probability distribution of future trajectories of long-term landscape evolution. Numbers in circles label calculation steps described in the text.

sults in probability distributions of change for a given landscape type to any other type. These probability distributions are calculated using a Mathematica (Wolfram Research, 2019 ) script. The script performs a simple tally of all transitions in our dataset (or in its subset).

Step 5. Assuming that transition probabilities (see step 4) calculated from 19922015 statistics are stationary, we simulate a future trajectory of a given landscape using a stochastic process. At the start of the simulation $\left(t_{s}\right)$ a landscape of a given type is considered. This type has a probability distribution $\left\{p_{1}, \ldots, p_{n}\right\}$ of transiting to another type during the simulation step $\Delta t$. As $\sum_{1}^{n} p_{i}=1$, we divide the interval $[0,1]$ into subintervals using the aforementioned probabilities; that is, the first subinterval is $\left(0, p_{1}\right]$, the second subinterval is $\left(p_{1}, p_{1}+p_{2}\right]$ etc. until the last subinterval is $\left(p_{1}+\ldots+\right.$ 
$p_{n-1}, p_{1}+\ldots+p_{n}=1$ ], where $n$ is the number of nonempty landscape types. We then draw a random number from an interval $[0,1]$ and choose a landscape type at simulation step $t_{s+1}$ corresponding to subinterval into which a drawn value fells. This is a classic Monte Carlo simulation procedure, the landscape type at the next step is selected randomly from a given distribution of transitions from the preceding landscape type. The subsequent steps of simulation follow the same rule. Calculations are performed using a Mathematica script.

Step 6. Starting from the same landscape type (Ad) of the homogeneous forest, we simulate 20,000 trajectories following the procedure described in Step 5. Next, we tally all trajectories to obtain frequencies of unique trajectories. These frequencies are converted into probabilities yielding a probability distribution function of future trajectories. 

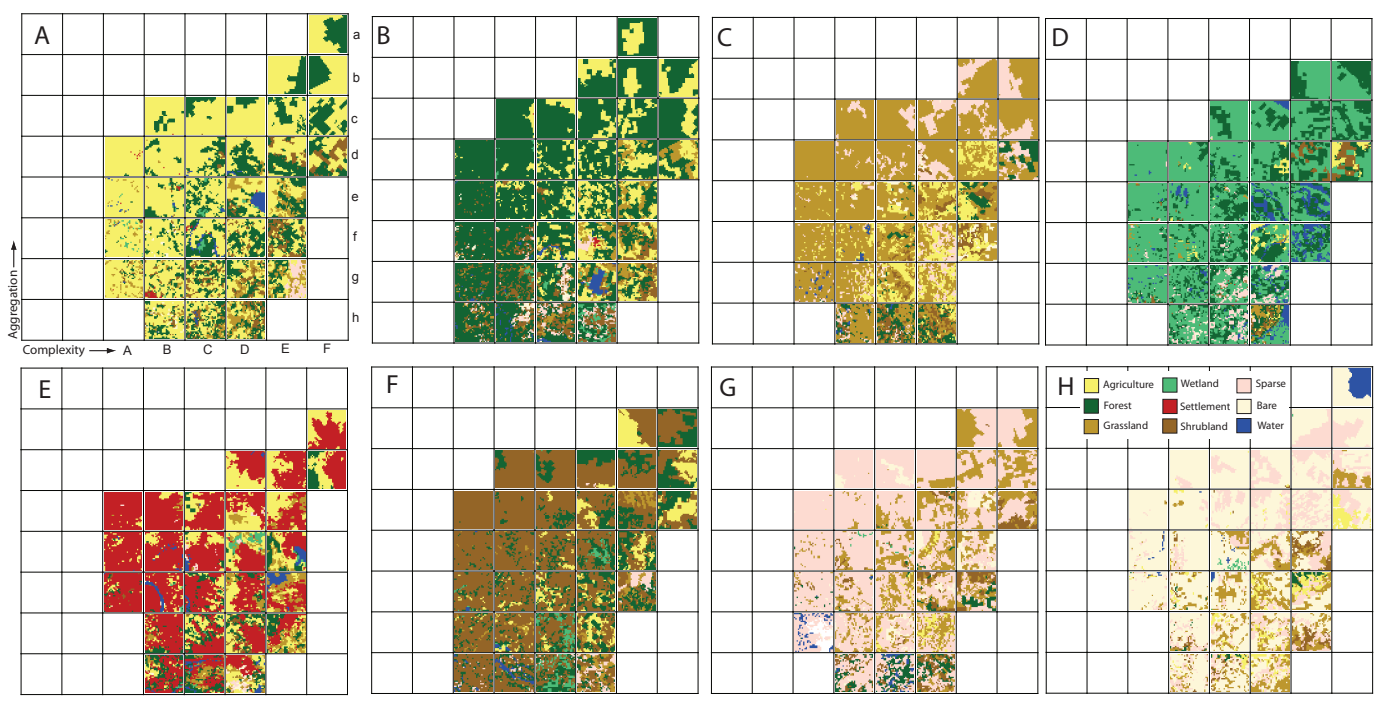

Figure S2. Archetypes of pattern configurations for segments of the $C \quad-\quad A$ diagram. Eight different panels (A to $\mathrm{H}$ ) indicate different thematic subsets of the landscapes' database. Archetypes are selected from this database based on what land cover class constitutes the largest share of the landscape area; (A) agriculture, (B) forest, (C) grassland, (D) wetland, (E) urban, (F) shrubland, (G) sparse vegetation, and (H) bare land. Empty segments in each $C-A$ diagram means that no landscapes of such configurations were present in this subset of landscapes. The secondary shares of landscape composition are not controlled, but they tend to be land cover classes that naturally form mosaics with the land cover class of the dominant share. 

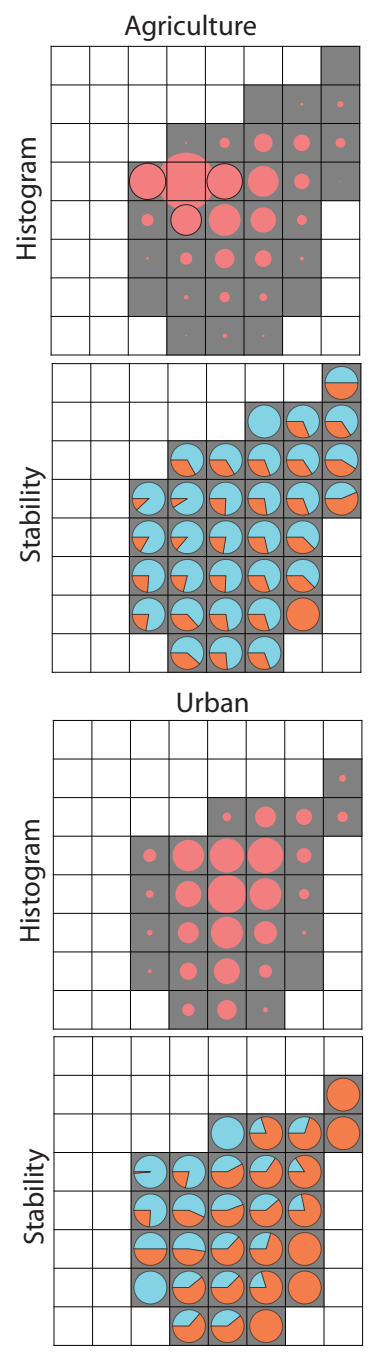

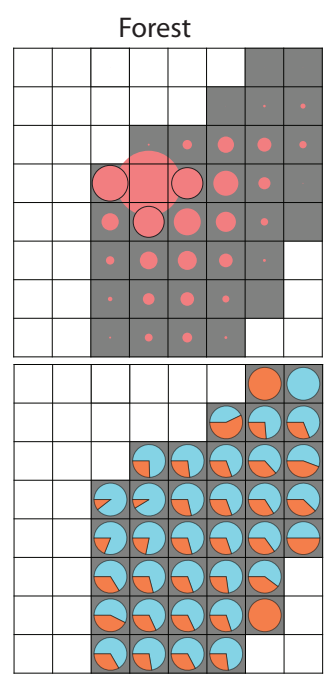

Shrubland

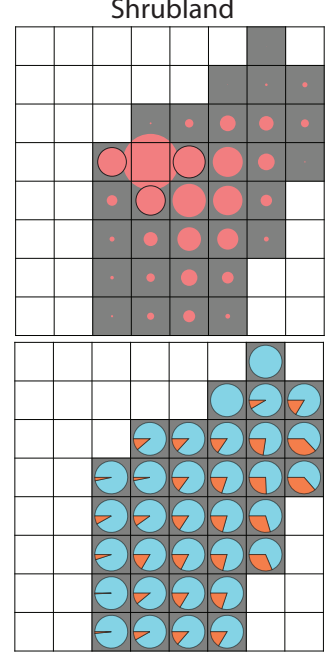

Grassland

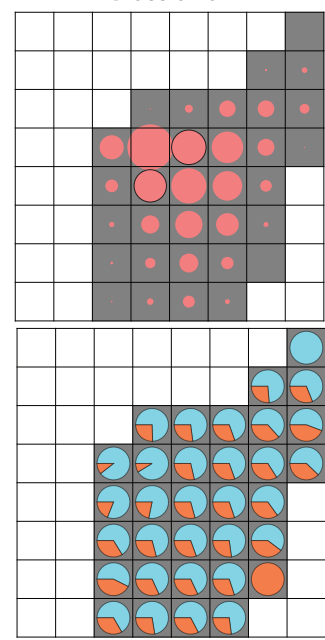

Sparse vegetation

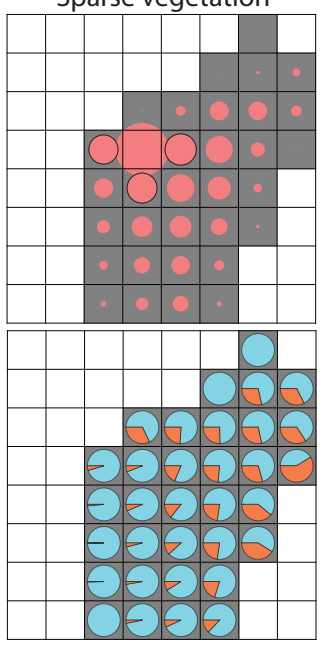

Wetland

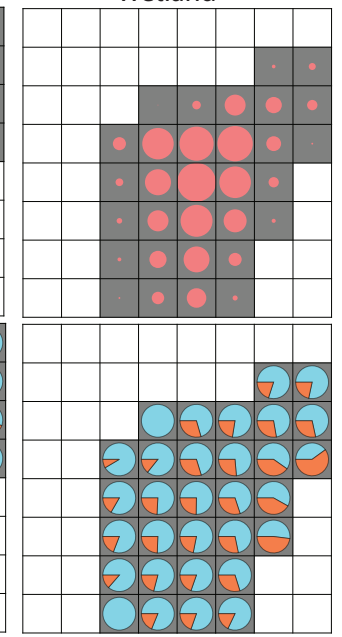

Bare land

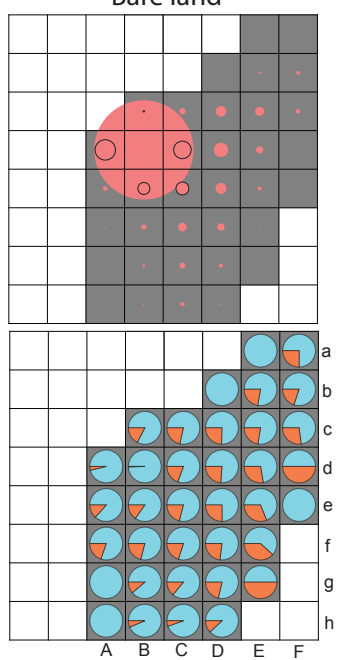

Figure S3. Statistics of thematic subsets of landscapes in the database selected based on a land cover class, which constitutes the largest share of the landscape area. Eight sections of the figure correspond to eight land cover classes as labeled. Each section has two panels. Top panels show histograms of landscapes in 1992 with bins corresponding to their pattern types (sectors of the $C \quad-\quad A$ diagram). Gray-colored sectors indicate non-zero bins. Areas of red circles are proportional to the fraction of landscapes in the bin; the sum of all bins equals to 1 . Bottom panels show probabilities of landscapes in a given sector of the $C-A$ diagram to remain in this sector (blue) or to transfer to another sector (red) during the period 1992-2015. The larger the "remain" (blue) part, the more stable the landscape configuration. Most configurations of urban landscapes are unstable due to urbanization. On the other hand, most configurations of sparse vegetation and bare lands landscapes are stable. 

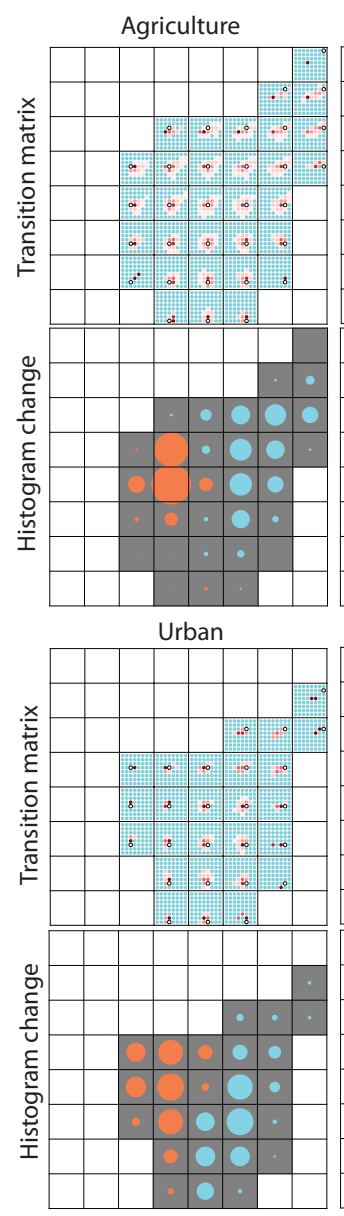

Forest

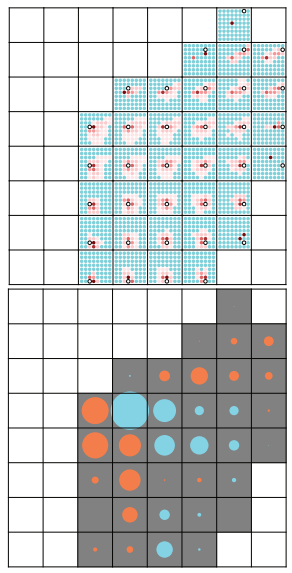

Shrubland

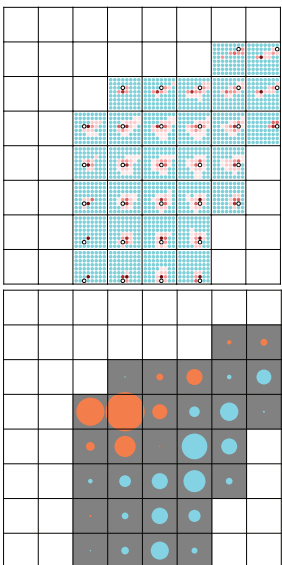

Grassland

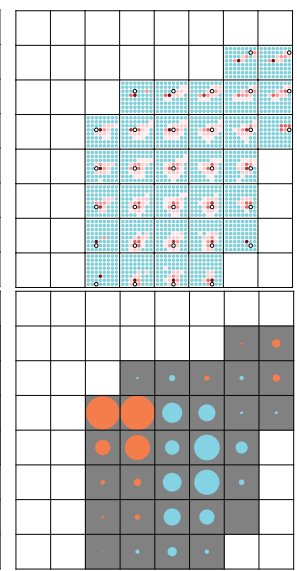

Sparse vegetation

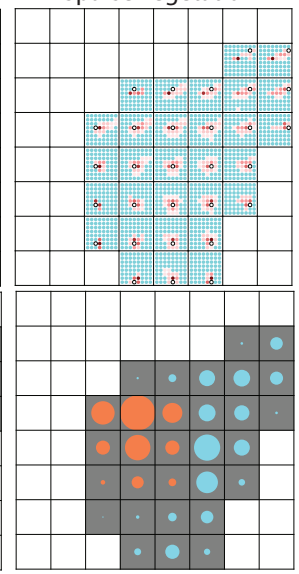

Wetland

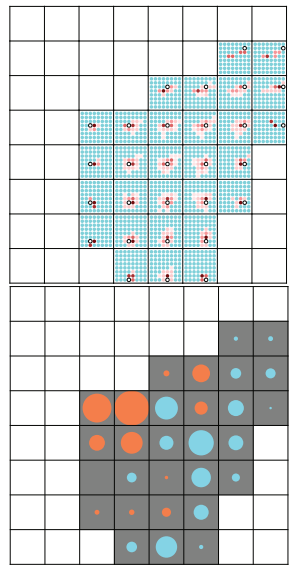

Bare land

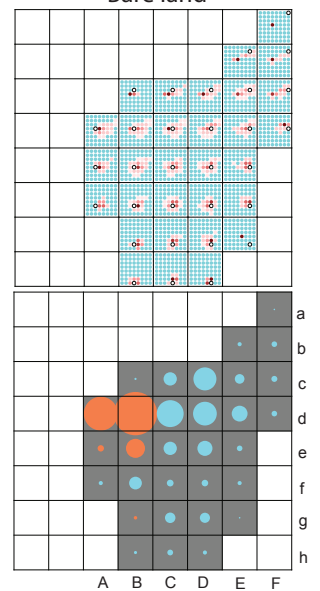

Figure S4. Visualizations of transition matrices for models built using subsets of landscapes in the database, as described in Figs. S1 and S2. Eight sections of the figure correspond to eight land cover classes as labeled. Each section has two panels. Top panels show a visualization of transition matrices. Each matrix is divided into smaller parts $(8 \times 8$ matrices $)$ corresponding to transitions from specific pattern types. These parts are organized in the $C-A$ grid. Each part itself is also organized in accordance with the $C-A$ diagram. Non-blue circles indicate pattern types to which a given landscape could transition during $\Delta t$, the darker the color the larger the probability of transition. Bottom panels show net changes to histograms of landscape configurational types during $\Delta t$. Areas of circles are proportional to the magnitude of the net change, red color indicates the gain and blue color indicates the loss. Note differences between the distribution of net gains and losses among different pattern types for different thematic subsets of the landscape database. 

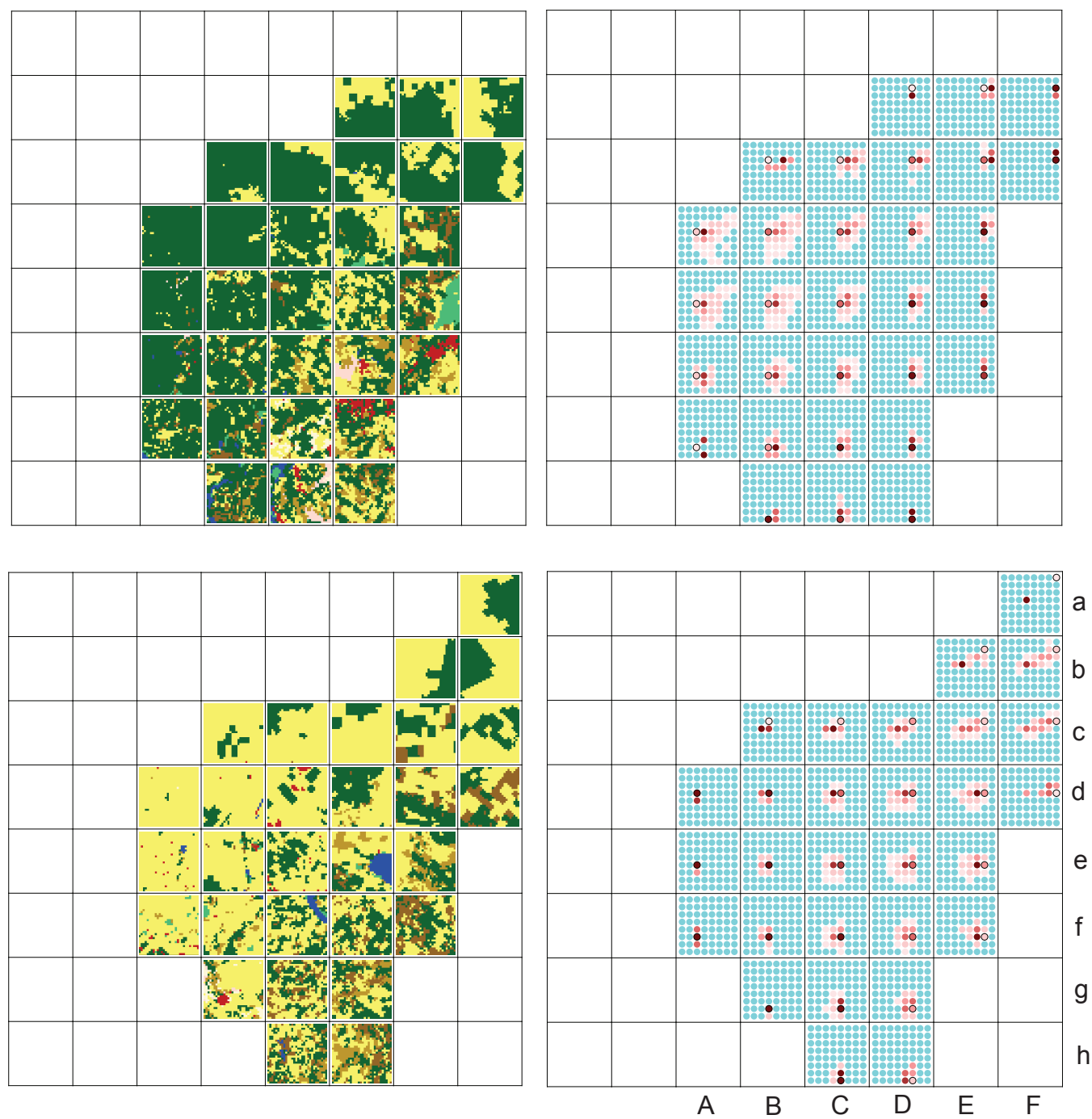

Figure S5. Models of phase 1 (top row) and phase 2 (bottom row) of the forest $\rightarrow$ agriculture transition. Left panels show archetypes of pattern configurations. Note that these are not the same as archetypes shown in Fig. S1 panels B and A because they were derived from different thematic subsets. Right panels are visualizations of models' transitions matrices, for details see a caption to Fig. S3. 


\section{References}

Netzel, P., Nowosad, J., Jasiewicz, J., Niesterowicz, J., \& Stepinski, T. F. February). GeoPAT 2: user's manual. Zenodo. Retrieved from https://doi.org/ 10.5281/zenodo. 1291123 doi: 10.5281/zenodo.1291123

Nowosad, J., \& Stepinski, T. F. (2018a). Global inventory of landscape patterns and latent variables of landscape spatial configuration. Ecological Indicators, 89, 159167.

Nowosad, J., \& Stepinski, T. F. (2018b). Global mapping of changes in landscapes and coverages of vegetation types from the ESA land cover 1992-2015 time series. EarthArXiv Preprints. Retrieved from https://eartharxiv.org/k3rmn/ doi: $10.31223 /$ osf.io/k3rmn

Nowosad, J., Stepinski, T. F., \& Netzel, P. (2019, Jan). Global assessment and mapping of changes in mesoscale landscapes: 1992-2015. OSF. Retrieved from osf .io/34g9c

R Core Team. (2019). R: A language and environment for statistical computing [Computer software manual]. Vienna, Austria. Retrieved from https://www.R -project.org/

Wolfram Research, I. (2019). Mathematica (12.0 ed.). Wolfram Research, Inc. Retrieved from http://www.wolfram.com/mathematica 